\title{
Hybrid approximations for fractional calculus
}

\author{
Mohsen Razzaghi ${ }^{1, *}$ \\ ${ }^{1}$ Department of Mathematics and Statistics, Mississippi State University, Mississippi State, MS 39762, \\ USA
}

\begin{abstract}
In this paper, a numerical method for solving the fractional differential equations is presented. The method is based upon hybrid functions approximation. The properties of hybrid functions consisting of block-pulse functions and Taylor polynomials are presented. The Riemann-Liouville fractional integral operator for hybrid functions is given. This operator is then utilized to reduce the solution of the initial value problems for fractional differential equations to a system of algebraic equations. Illustrative examples are included to demonstrate the validity and applicability of the technique.
\end{abstract}

Keywords: Hybrid functions; fractional differential equations; block-pulse; Taylor polynomials; Caputo derivative; numerical solution

\section{Introduction}

Fractional differential equations (FDEs) are generalized from integer order ones, which are obtained by replacing integer order derivatives by fractional ones. Many physical systems are modeled using fractional differential equations (FDEs). (see for example [1-4] and references therein).

Due to the extensive applications of FDEs in engineering and science, research in this area has grown significantly, and there has been considerable interest in developing numerical schemes for their solution. These methods include Fourier transforms [5], eigenvector expansion [6], Laplace transforms [7], Adomian decomposition method [8], variational iteration method [9], finite difference method (FDM) [10], the power series method [11], fractional differential transform method (FDTM) [12], and homotopy analysis method [13].

The available sets of orthogonal functions can be divided into three classes. The first class includes sets of piecewise constant basis functions (e.g., block-pulse, Haar, Walsh, etc.). The second class consists of sets of orthogonal polynomials (e.g., Chebyshev, Laguerre, Legendre, etc.). The third class is the set of sine-cosine functions in the Fourier series. Orthogonal functions have been used when dealing with various problems of the dynamical systems. The main advantage of using orthogonal functions is that they reduce the dynamical system problems to those of solving a system of algebraic equations by using the operational matrices of differentiation or integration. These matrices can be uniquely determined based on the particular orthogonal functions. Special attention has been given to applications of the Walsh functions, rationalized Haar functions and Legendre wavelets [14-16]. The Bernoulli polynomials and Taylor series are not based on orthogonal functions. Nevertheless, they possess the operational matrix of integration.

\footnotetext{
*e-mail: razzaghi@math.msstate.edu
} 
In recent years, the hybrid functions consisting of the combination of block-pulse functions with Legendre polynomials, Chebyshev polynomials, Taylor series, or Bernoulli polynomials [17-20] have been successfully used for solving selected smooth and non-smooth problems arising in diverse areas of science and engineering. Among the hybrid functions, the structures of the hybrid of block-pulse functions and Taylor polynomials are much simpler than the other hybrid functions [21]. The novel contributions for using the hybrid of block-pulse functions and Taylor polynomials together with their error analysis are given in [21]. In 2015, to the best of our knowledge, the Riemann-Liouville fractional integral operator for hybrid of block-pulse functions and Bernoulli polynomials was derived directly for the first time $[22,23]$.

In the present paper, a new numerical method for solving the initial value problem for fractional order differential equations is presented. The method is based upon hybrid functions approximation. These hybrid functions, which consist of block-pulse functions and Taylor polynomials, are included. An exact Riemann-Liouville fractional integral operator for the hybrid of block-pulse functions and Taylor polynomials is given. This operator is then utilized to reduce the solution of the fractional order differential equations to the solution of algebraic equations.

\section{Preliminaries and notations}

\subsection{The fractional integral and derivative}

Definition 1 The Riemann-Liouville fractional integral operator of order $\alpha$ is defined as [2]

$$
I^{\alpha} f(t)= \begin{cases}\frac{1}{\Gamma(\alpha)} \int_{0}^{t} \frac{f(s)}{(t-s)^{1-\alpha}} d s=\frac{1}{\Gamma(\alpha)} t^{\alpha-1} * f(t), & \alpha>0 \\ f(t), & \alpha=0\end{cases}
$$

where $t^{\alpha-1} * f(t)$ is the convolution product of $t^{\alpha-1}$ and $f(t)$.

For the Riemann-Liouville fractional integral, we have

$$
I^{\alpha} t^{v}=\frac{\Gamma(v+1)}{\Gamma(v+1+\alpha)} t^{\nu+\alpha}, \quad v>-1 .
$$

Definition 2 Caputo's fractional derivative of order $\alpha$ is defined as [2]

$$
\left(D^{\alpha} f\right)(t)=\frac{1}{\Gamma(n-\alpha)} \int_{0}^{t} \frac{f^{(n)}(s)}{(t-s)^{\alpha+1-n}} d s, \quad n-1<\alpha \leq n, n \in \mathbb{N},
$$

where $\alpha>0$ is the order of the derivative, and $n$ is the smallest integer greater than $\alpha$.

The Riemann-Liouville integral operator and Caputo derivative satisfy the following properties [2]

$$
I^{\alpha}\left(D^{\alpha} h(t)\right)=h(t)-\sum_{k=0}^{n-1} h^{(k)}(0) \frac{t^{k}}{k !},
$$

if $\alpha \in \mathbb{R}, n-1<\alpha \leq n, n \in \mathbb{N}$, then

$$
D^{\alpha} h(t)=I^{n-\alpha} D^{n} h(t)
$$

Also, for $\alpha \geq \beta$ we have

$$
D^{\beta} B(t, \alpha)=B(t, \alpha-\beta)
$$




\section{Properties of hybrid functions}

\subsection{Hybrid block-pulse functions and Taylor polynomials}

Hybrid functions $b_{n m}(t), n=1,2, \ldots, N, m=0,1, \ldots, M$ are defined on the interval $\left[0, t_{f}\right)$ as [22]

$$
b_{n m}(t)=\left\{\begin{array}{cl}
T_{m}\left(N t-(n-1) t_{f}\right), & t \in\left[\frac{n-1}{N} t_{f}, \frac{n}{N} t_{f}\right), \\
0, & \text { otherwise, }
\end{array}\right.
$$

where $n$ denotes the order of block-pulse functions. In Eq. (4), $T_{m}(t)=t^{m}, m=0,1, \ldots, M$ are the well-known Taylor polynomials of order $\mathrm{m}$. It should be pointed out that the hybrid of block-pulse functions and Taylor polynomials constitutes a semi-orthogonal set on the interval $\left[0, t_{f}\right)$. This fact is due to the disjointedness property of block-pulse functions. According to Eq. (4), a set of six elements of these functions corresponding to $N=2, M=2$, and $t_{f}=1$, are expressed by

$$
\left.\left.\begin{array}{l}
b_{10}(t)=T_{0}(2 t)=1 \\
b_{11}(t)=T_{1}(2 t)=2 t \\
b_{12}(t)=T_{2}(2 t)=4 t^{2}
\end{array}\right\} 0 \leq t<\frac{1}{2}, \quad \begin{array}{l}
b_{20}(t)=T_{0}(2 t-1)=1 \\
b_{21}(t)=T_{1}(2 t-1)=2 t-1 \\
b_{22}(t)=T_{2}(2 t-1)=(2 t-1)^{2}
\end{array}\right\} \frac{1}{2} \leq t<1 .
$$

\subsection{Function approximation}

A function $f(t)$ defined over the interval $\left[0, t_{f}\right)$ may be expanded as

$$
f(t)=\sum_{n=1}^{\infty} \sum_{m=0}^{\infty} c_{n m} b_{n m}(t)
$$

where

$$
c_{n m}=\left.\frac{1}{N^{m} m !}\left(\frac{d^{m} f(t)}{d t^{m}}\right)\right|_{t=\left(\frac{n-1}{N}\right) t_{f}} .
$$

If the infinite series in Eq. (5) is truncated, then Eq. (5) can be written as

$$
f(t) \simeq \sum_{n=1}^{N} \sum_{m=0}^{M} c_{n m} b_{n m}(t)=C^{T} B(t),
$$

where

$$
C^{T}=\left[c_{10}, c_{20}, \ldots, c_{N 0}, c_{11}, c_{21}, \ldots, c_{N 1}, \ldots, c_{1 M}, c_{2 M}, \ldots, c_{N M}\right]
$$

and

$$
B^{T}(t)=\left[b_{10}(t), \ldots, b_{N 0}(t), b_{11}(t), \ldots, b_{N 1}(t), \ldots, b_{1 M}(t), \ldots, b_{N M}(t)\right]
$$

\section{Riemann-Liouville fractional integral operator for hybrid of block-pulse functions and Taylor series}

We now derive the operator $I^{\alpha}$ for $B(t)$ in Eq. (6) given by

$$
I^{\alpha} B(t)=\bar{B}(t, \alpha)
$$


where

$$
\bar{B}(t, \alpha)=\left[I^{\alpha} b_{10}(t), \ldots, I^{\alpha} b_{N 0}(t), I^{\alpha} b_{11}(t), \ldots, I^{\alpha} b_{N 1}(t), \ldots, I^{\alpha} b_{1 M}(t), \ldots, I^{\alpha} b_{N M}(t)\right]^{T} .
$$

To obtain $I^{\alpha} b_{n m}(t)$, we use the Laplace transform. By using Eq. (4) with $t \in\left[0, t_{f}\right)$, we have

$$
b_{n m}(t)=\mu_{\frac{n-1}{N} t_{f}}(t) T_{m}\left(N t-(n-1) t_{f}\right)-\mu_{\frac{n}{N} t_{f}}(t) T_{m}\left(N t-(n-1) t_{f}\right),
$$

where $\mu_{c}(t)$ is the unit step function defined as

$$
\mu_{c}(t)= \begin{cases}1, & t \geq c \\ 0, & t<c .\end{cases}
$$

By taking the Laplace transform from Eq. (8) and using

$$
\mathscr{L}\left\{\mu_{c}(t) f(t-c)\right\}=e^{-c s} \mathscr{L}\{f(t)\},
$$

we get

$$
\begin{aligned}
\mathscr{L}\left\{b_{n m}(t)\right\}= & \mathscr{L}\left\{\mu_{\frac{n-1}{N} t_{f}}(t) T_{m}\left(N\left(t-\frac{n-1}{N} t_{f}\right)\right)\right\} \\
& -\mathscr{L}\left\{\mu_{\frac{n}{N}}(t) T_{m}\left(N\left(t-\frac{n}{N} t_{f}\right)+t_{f}\right)\right\} \\
= & e^{-\frac{n-1}{N} t_{f} s} \mathscr{L}\left\{T_{m}(N t)\right\}-e^{-\frac{n}{N} t_{f} s} \mathscr{L}\left\{T_{m}\left(N t+t_{f}\right)\right\} \\
= & \frac{N^{m} e^{-\frac{n-1}{N} t_{f} s} m !}{s^{m+1}}-\sum_{k=0}^{m} \frac{N^{k} e^{-\frac{n}{N} t_{f} s}\left(\begin{array}{c}
m \\
k
\end{array}\right) k ! t_{f}^{m-k}}{s^{k+1}} .
\end{aligned}
$$

From Eq. (1) we get

$$
\begin{aligned}
\mathscr{L}\left\{I^{\alpha} b_{n m}(t)\right\} & =\frac{1}{\Gamma(\alpha)} \frac{\Gamma(\alpha)}{s^{\alpha}}\left[\mathscr{L}\left\{b_{n m}(t)\right\}\right] \\
& =\frac{N^{m} e^{-\frac{n-1}{N} t_{f} s} m !}{s^{m+1+\alpha}}-\sum_{k=0}^{m} \frac{N^{k} e^{-\frac{n}{N} t_{f} s}\left(\begin{array}{c}
m \\
k
\end{array}\right) k ! t_{f}^{m-k}}{s^{k+1+\alpha}} .
\end{aligned}
$$

Taking the inverse Laplace transform of Eq. (9) yields

$$
I^{\alpha} b_{n m}(t)=N^{m} m ! \mu_{\frac{n-1}{N} t_{f}}(t) \frac{\left(t-\frac{n-1}{N} t_{f}\right)^{m+\alpha}}{\Gamma(m+1+\alpha)}-\sum_{k=0}^{m} N^{k}\left(\begin{array}{c}
m \\
k
\end{array}\right) k ! t_{f}^{m-k} \mu_{\frac{n}{N} t_{f}}(t) \frac{\left(t-\frac{n}{N} t_{f}\right)^{k+\alpha}}{\Gamma(k+1+\alpha)}
$$

By using Eq. (10), we have

$$
I^{\alpha} b_{n m}(t)=\left\{\begin{array}{cc}
0, & t \in\left[0, \frac{n-1}{N} t_{f}\right), \\
N^{m} m ! \frac{\left(t-\frac{n-1}{N} t_{f}\right)^{m+\alpha}}{\Gamma(m+1+\alpha)}, & t \in\left[\frac{n-1}{N} t_{f}, \frac{n}{N} t_{f}\right), \\
N^{m} m ! \frac{\left(t-\frac{n-1}{N} t_{f}\right)^{m+\alpha}}{\Gamma(m+1+\alpha)}-\sum_{k=0}^{m} N^{k}\left(\begin{array}{c}
m \\
k
\end{array}\right) k ! t_{f}^{m-k} \frac{\left(t-\frac{n}{N} t_{f}\right)^{k+\alpha}}{\Gamma(k+1+\alpha)}, & t \in\left[\frac{n}{N} t_{f}, t_{f}\right) .
\end{array}\right.
$$




\section{Problem statement}

We focus on the following problem:

Caputo fractional differential equation

$$
D^{\alpha} f(t)=h\left(t, f(t), D^{\beta} f(t)\right), \quad 1<\alpha \leq 2, \quad 0 \leq \beta \leq 1,
$$

with the initial conditions

$$
f(0)=f_{0}, \quad f^{\prime}(0)=f_{1}
$$

\section{Numerical method}

In this section, we use the hybrid of block-pulse functions and Taylor polynomials for solving Eq. (11) with the initial conditions in Eq. (12).

In this case, we expand $D^{\alpha} f(t)$ by the hybrid functions as

$$
D^{\alpha} f(t)=A^{T} B(t)
$$

using Eq. (2) and (13), we obtain

$$
f(t)=A^{T} B(t, \alpha)+f^{\prime}(0) t+f(0)
$$

from Eq. (14), we get

$$
D^{\beta} f(t)=A^{T} \bar{B}(t, \alpha-\beta)+f^{\prime}(0) \frac{\Gamma(2)}{\Gamma(2-\beta)} t^{1-\beta},
$$

Substituting Eqs. (13)-(15) in Eq. (11), we get a system of algebraic equations. Next, we collocate these equations at the Newton-cotes nodes $t_{i}$ given by

$$
t_{i}=\frac{i+1}{2 N(M+1)}, \quad i=0,1, \ldots, 2 N(M+1)-2 .
$$

These equations give $N(M+1)$ algebraic equations, which can be solved for the unknown vector $A^{T}$ using Newton's iterative method.

\section{Illustrative Example}

In this section two examples are given to demonstrate the applicability and accuracy of our method.

\subsection{Example 1}

Consider the equation [24]

$$
D^{2} f(t)+3 D f(t)+2 D^{q_{2}} f(t)+D^{q_{1}} f(t)+5 f(t)=g(t), \quad 0<q_{1}<q_{2}<1,
$$

with

$$
g(t)=1+3 t+\frac{2}{\Gamma\left(3-q_{2}\right)} t^{2-q_{2}}+\frac{1}{\Gamma\left(3-q_{1}\right)} t^{2-q_{1}}+5\left(1+\frac{1}{2} t^{2}\right)
$$

subject to

$$
f(0)=1, \quad f^{\prime}(0)=0,
$$


where $q_{1}=0.0159$ and $q_{2}=0.1379$. The exact solution of this problem is [24]

$$
f(t)=1+\frac{1}{2} t^{2}
$$

Here, we solve this problem by using the Hybrid functions with $N=1$ and $M=2$. Let

$$
D^{2} f(t)=A^{T} B(t)=\left[a_{10}, a_{11}, a_{12}\right]\left[\begin{array}{l}
b_{10}(t) \\
b_{11}(t) \\
b_{12}(t)
\end{array}\right] .
$$

Then, by using Eqs. (7) and(19), we have

$$
\begin{gathered}
f(t)=A^{T} \bar{B}(t, 2)+1, \quad D f(t)=A^{T} \bar{B}(t, 1), \\
D^{q_{2}} f(t)=A^{T} \bar{B}\left(t, 2-q_{2}\right), \quad D^{q_{1}} f(t)=A^{T} \bar{B}\left(t, 2-q_{1}\right),
\end{gathered}
$$

By substituting Eqs. (19) and (21) in Eq. (17), we get

$$
A^{T} B(t)+3 A^{T} \bar{B}(t, 1)+2 A^{T} \bar{B}\left(t, 2-q_{2}\right)+A^{T} \bar{B}\left(t, 2-q_{1}\right)+5\left(A^{T} \bar{B}(t, 2)+1\right)=g(t) .
$$

By collocating Eq. (22) at the Newton-cotes nodes given in Eq. (16), we get

$$
f(t)=1+\frac{1}{2} t^{2}
$$

which is the exact solution.

\subsection{Example 2}

Consider fractional Riccati equation [25]

$$
D^{q} f(t)=2 f(t)-f^{2}(t)+1, \quad 0<q \leq 1,
$$

subject to the initial state $f(0)=0$. To solve by using the present method, we let

$$
D^{q} f(t)=A^{T} B(t)
$$

from Eqs. (7) and (24), we get

$$
f(t)=A^{T} \bar{B}(t, q)
$$

By substituting Eqs. (24) and (25) in Eq. (23) and collocating at the Newton-cotes nodes given in Eq. (16), we get $N(M+1)$ nonlinear algebraic equations which can be solved for the unknown vector $A$ using Newton's iterative method. The exact solution of this problem, when $q=1$, is

$$
f(t)=1+\sqrt{2} \tanh \left(\sqrt{2} t+\frac{1}{2} \ln \left(\frac{\sqrt{2}-1}{\sqrt{2}+1}\right)\right) .
$$

The numerical results for $f(t)$ with $N=1, M=5$, and $q=0.7,0.8,0.9,1$ are plotted in Figure 1. The approximate solutions, using the present method, are in agreement with the exact solutions for $q=1$ and, as indicated in Figure 2, the solution continuously depends on the time-fractional derivative. 


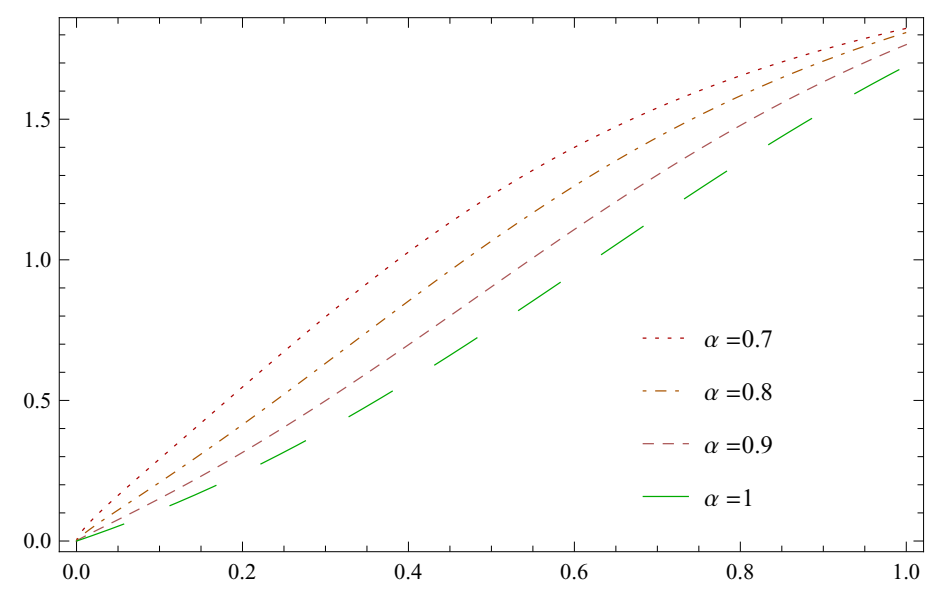

Figure 1. Comparison of $\mathrm{f}(\mathrm{t})$ for $\mathrm{N}=1, \mathrm{M}=5$, with $q=0.7,0.8,0.9$ and exact solution

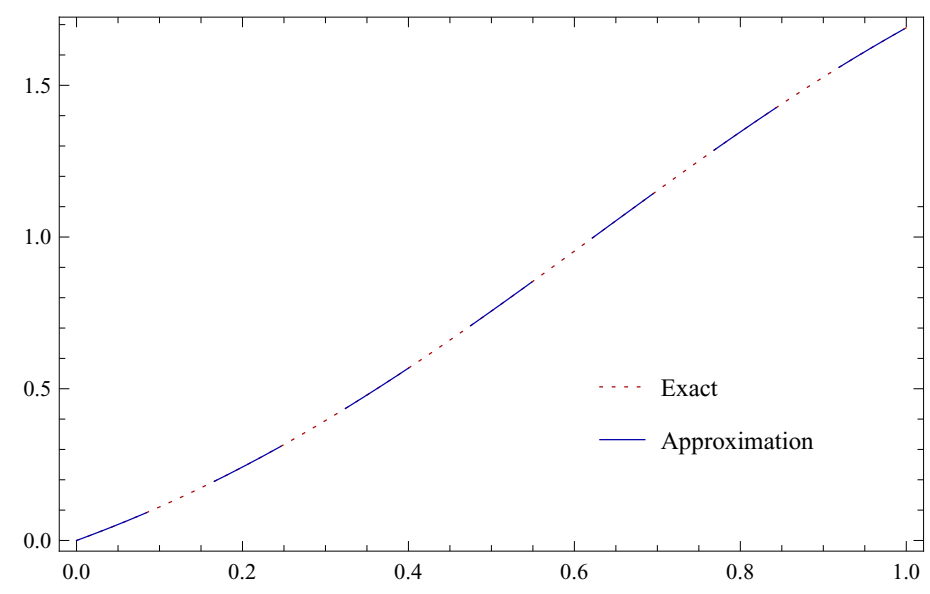

Figure 2. Plot of $\mathrm{f}(\mathrm{t})$ obtained from present method with $\mathrm{N}=1, \mathrm{M}=5$, and exact solution

\section{Conclusion}

A general formulation for the Riemann-Liouville fractional integral operator for hybrid of block-pulse functions and Taylor polynomials has been derived. This operator is used to approximate numerical solution of FDEs. In the limit, as $q$ approaches an integer value, the scheme provides solution for the integer-order differential equations. The solution obtained using the present method shows that this approach can solve the problem effectively.

\section{References}

[1] Oldham K.B, Spanier J. The Fractional Calculus, Academic Press: New York. 1974.

[2] Miller K.S, Ross B. An Introduction to the Fractional Calculus and Fractional Differential Equations, Wiley: New York. 1993.

[3] Bagley R.L, Torvik P.J. Fractional calculus in the transient analysis of viscoelastically damped structures, AIAA J. 1985; 23: 918-925. 
[4] Chow T.S. Fractional dynamics of interfaces between soft-nanoparticles and rough substrates, Phys. Lett. A. 2005; 342: 148-155.

[5] Gaul L, Klein P, Kemple S. Damping description involving fractional operators, Mech. Syst. Signal. Pr. 1991; 5: 81-88.

[6] Suarez L, Shokooh A. An eigenvector expansion method for the solution of motion containing fractional derivatives, J. Appl. Mech. 1997; 64: 629-735.

[7] Podlubny I. Fractional Differential Equations: An Introduction to Fractional Derivatives, Fractional Differential Equations, to Methods of Their Solution and Some of Their Applications, Academic Press, New York. 1998.

[8] Momani S, Al-Khaled K. Numerical solutions for systems of fractional differential equations by the decomposition method, Appl. Math. Comput. 2005; 162: 1351-1365.

[9] Odibat Z, Momani S. Application of variational iteration method to nonlinear differential equations of fractional order, Int. J. Nonlinear Sci. Numer. Simul. 2006; 7: 27-34.

[10] Meerschaert M, Tadjeran C. Finite difference approximations for two-sided spacefractional partial differential equations, Appl. Numer. Math. 2006; 56: 80-90.

[11] Odibat Z, Shawagfeh N. Generalized Taylor's formula, Appl. Math. Comput. 2007; 186: 286-293.

[12] Arikoglu A, Ozkol I. Solution of fractional integro-differential equations by using fractional differential transform method, Chaos Solitons Fract 2009; 40: 521-529.

[13] Hashim I, Abdulaziz O, Momani S. Homotopy analysis method for fractional IVPs, Commun. Nonlinear Sci. Numer. Simul. 2009; 14: 674-684.

[14] Chen C.F, Hsiao C.H. A Walsh series direct method for solving variational problems, J. Frank. Inst. 1975; 300: 265-280.

[15] Razzaghi M, Ordokhani Y. An application of rationalized Haar functions for variational problems, Appl. Math. Comput. 2001; 122: 353-364.

[16] Razzaghi M, Yousefi S. Legendre wavelets method for the solution of nonlinear problems in the calculus of variations, Math. Comput. Model. 2001; 34: 45-54.

[17] Razzaghi M, Marzban H. Hybrid analysis direct method in the calculus of variations, Int. J. Comput. Math. 2000; 75: 259-269.

[18] Razzaghi M, Marzban H. Direct method for variational problems via hybrid of blockpulse and Chebyshev functions, Math. Prob. Eng. 2000; 6: 85-97.

[19] Marzban H, Razzaghi M. Analysis of time-delay systems via hybrid of block-pulse functions and Taylor series, J. Vibr. Contr. 2005; 11: 1455-1468.

[20] Haddadi N, Ordokhani Y, Razzaghi M. Optimal Control of Delay Systems by Using a Hybrid Functions Approximation, J. Optimi. Theo. Appli. 2012; 153: 338-356.

[21] Marzban, H. R., 2016, "Parameter identification of linear multi-delay systems via a hybrid of block-pulse functions and Taylors polynomials," Int. J. Control., 1, pp. 1-15.

[22] Mashayekhi, S., and Razzaghi, M., 2015, "Numerical solution of nonlinear fractional integro-differential equations by hybrid functions," Eng. Analysis Bound. Elem., 56, pp. $81-89$.

[23] Mashayekhi, S., and Razzaghi, M., 2016, "Numerical solution of distributed order fractional differential equations by hybrid functions," J. Comput. Physics., 315, pp. 169-181.

[24] El-Sayed A.M.A, El-Mesiry A.E.M, El-Saka HAA. Numerical solution for multi-term fractional (arbitrary) orders differential equations, Comput. appl. Math. 2004; 23: 33-54.

[25] Odibat Z, Momani S. Modified homotopy perturbation method: application to quadratic Riccati differential equation of fractional order, Chaos. Solitons. Fract. 2008; 36: 167-174. 